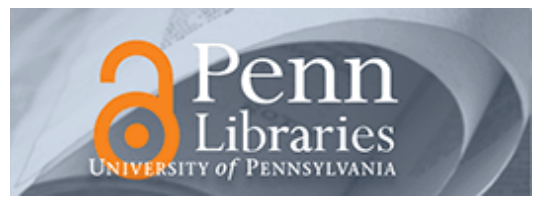

University of Pennsylvania

ScholarlyCommons

Statistics Papers

Wharton Faculty Research

$10-2014$

\title{
Minimum Enclosing Circle of a Set of Fixed Points and a Mobile Point
}

Aritra Banik

Bhaswar B. Bhattacharya

University of Pennsylvania

Sandip Das

Follow this and additional works at: https://repository.upenn.edu/statistics_papers

Part of the Applied Mathematics Commons, Business Analytics Commons, Management Sciences and Quantitative Methods Commons, Mathematics Commons, and the Statistics and Probability Commons

\section{Recommended Citation}

Banik, A., Bhattacharya, B. B., \& Das, S. (2014). Minimum Enclosing Circle of a Set of Fixed Points and a Mobile Point. Computational Geometry, 47 (9), 891-898. http://dx.doi.org/10.1016/j.comgeo.2014.04.006

This paper is posted at ScholarlyCommons. https://repository.upenn.edu/statistics_papers/648

For more information, please contact repository@pobox.upenn.edu. 


\title{
Minimum Enclosing Circle of a Set of Fixed Points and a Mobile Point
}

\author{
Abstract \\ Given a set $S$ of $n$ static points and a mobile point $p$ in $\mathbb{R}^{2}$, we study the variations of the smallest circle \\ that encloses $S \cup\{p\}$ when $p$ moves along a straight line $\ell$. In this work, a complete characterization of \\ the locus of the center of the minimum enclosing circle (MEC) of $S \cup\{p\}$, for $p \in \ell$, is presented. The \\ locus is a continuous and piecewise differentiable linear function, and each of its differentiable pieces lies \\ either on the edges of the farthest-point Voronoi diagram of $S$, or on a line segment parallel to the line $\ell$. \\ Moreover, the locus has differentiable pieces, which can be computed in linear time, given the farthest- \\ point Voronoi diagram of $S$.

\section{Keywords} \\ farthest-point Voronoi diagram, minimum enclosing circle, mobile facility location

\section{Disciplines} \\ Applied Mathematics | Business | Business Analytics | Management Sciences and Quantitative Methods | \\ Mathematics | Statistics and Probability
}




\title{
Minimum Enclosing Circle of a Set of Fixed Points and a Mobile Point*
}

\author{
Aritra Banik $^{1}$, Bhaswar B. Bhattacharya ${ }^{2}$, and Sandip Das ${ }^{1}$ \\ ${ }^{1}$ Advanced Computing and Microelectronics Unit, Indian Statistical Institute, \\ Kolkata - 700108, India, \\ aritrabanik@gmail.com, sandipdas@isical.ac.in \\ ${ }^{2}$ Department of Statistics, Stanford University, California, USA, bhaswar@stanford.edu
}

\begin{abstract}
Given a set $S$ of $n$ static points and a mobile point $p$ in $\mathbb{R}^{2}$, we study the variations of the smallest circle that encloses $S \cup\{p\}$ when $p$ moves along a straight line $\ell$. In this work, a complete characterization of the locus of the center of the minimum enclosing circle (MEC) of $S \cup\{p\}$, for $p \in \ell$, is presented. The locus is a continuous and piecewise differentiable linear function, and each of its differentiable pieces lies either on the edges of the farthest-point Voronoi diagram of $S$, or on a line segment parallel to the line $\ell$. Moreover, the locus has $O(n)$ differentiable pieces, which can be computed in linear time, given the farthest-point Voronoi diagram of $S$.
\end{abstract}

\section{Introduction}

Many 2D facility location problems mandate that a facility be placed amidst a set of users in the plane such that the maximum distance of the facility from the users is minimized. When the distance between a pair of points is measured in the $L_{2}$ metric (Euclidean metric), this gives rise to the notion of the Euclidean 1-center and the minimum enclosing circle (MEC) of a set of points. The Euclidean 1-center of a set of fixed points $S$ is the center of the smallest circle that encloses all the points of $S$. More formally, if the Euclidean distance between any two points $a$ and $b$ in $\mathbb{R}^{2}$ is denoted by $d(a, b)$, then for a finite set $S$ in $\mathbb{R}^{2}$, the Euclidean 1-center of $S$ is the point $\mathcal{E}(S)$ in $\mathbb{R}^{2}$ that minimizes the function $\lambda(q)=\max _{p_{i} \in S} d\left(p_{i}, q\right)$ over all points $q \in \mathbb{R}^{2}$. The value of $\lambda(\mathcal{E}(S))$ is the radius of the MEC of $S$ and is denoted by $r(S)$. These definitions can be naturally extended to higher dimensions as well.

It is believed that the question of finding the smallest circle enclosing a triangle was first posed by Archimedes [9]. However, the general question of finding the MEC of a set of $n$ points in the plane was first posed by Sylvester in 1857 [12]. Thereafter, several algorithms for computing the MEC of a set of $n$ points in the plane have been proposed. Finally, Megiddo [11] gave a deterministic $\Theta(n)$ time linear programming solution for the problem in $\mathbb{R}^{2}$. This result was extended to $\mathbb{R}^{d}$ in $O\left(d^{O(d)} n\right)$ time by Agarwal et al. [2] and Chazelle and Matoušek [7], and this is asymptotically optimal when $d$ is fixed. A simpler randomized algorithm with $O(n)$ expected time in $\mathbb{R}^{d}$ for any fixed $d$, was proposed by Welzl [13].

Motivated by recent advances in mobile computing, telecommunication, navigation, and geographic information system these questions have been posed in the mobile setting, where the points in the plane, which correspond to the users, move along some trajectories (see Durocher [9] for a survey on the various applications of mobile facility location problems). The problem of studying the variations of the Euclidean 1-median under continuous motion of points was initiated by Bereg et al. [4,5]. They showed that for any $v \geq 0$ there is a set of three points $s_{1}, s_{2}, s_{3}$ in $\mathbb{R}^{d}$ with $d \geq 2$, such that a unit velocity motion of two of the

\footnotetext{
* The conference version of this paper has appeared in the Proc. 5th International Workshop on Algorithms and Computation (WALCOM) 2011, 98-109.
} 
points induces an instantaneous velocity greater than $v$ of the Euclidean 1-center, i.e., the velocity of the mobile Euclidean 1-center is unbounded in $\mathbb{R}^{d}$ for $d \geq 2$. This motivated the problem of approximating the Euclidean 1-center by other center functions having bounded velocities [9]. Durocher also showed that under bounded continuous motion of the points in the plane, the Euclidean 1-center moves continuously in $\mathbb{R}^{d}$, for $d \geq 1$. Recently, Bitner et al. [3] studied the minimum separating circle problem, which is a bi-chromatic generalization of the MEC problem. Kinetic variantions of this problem were later studied by Cheung et al. [8].

In this paper, we provide a complete characterization of the locus of the Euclidean 1center for a set $S$ of $n$ fixed points and one mobile point moving along a straight line $\ell$. Choosing a coordinate system such that the line $\ell$ coincides with the $x$-axis, we define the center function $\psi: \mathbb{R} \rightarrow \mathbb{R}^{2}$, where $\psi(p)$ denotes the center of the MEC of $S \cup\{p\}$ in $\mathbb{R}^{2}$, for any point $p=(p, 0)$ on $\ell$. We show that the center function $\psi$ is a continuous and piecewise differentiable linear function, and each of its differentiable pieces lies either on the boundary of the farthest-point Voronoi diagram of $S$, or on a line parallel to the line $\ell$. Moreover, we prove that the combinatorial complexity of $\psi$, that is, number of the differentiable pieces in $\psi$ is $O(n)$. Based on this result, we give a simple $O(n)$ time algorithm for computing $\psi$, given the farthest-point Voronoi diagram of $S$.

Associated with the center function, one can define a radius function $\vartheta: \mathbb{R} \rightarrow \mathbb{R}$, where $\vartheta(p)$ is the radius of the MEC of $S \cup\{p\}$, for $p=(p, 0) \in \ell$. We show that if the line $\ell$ does not intersect the MEC of $S$, then there exits a point $p^{\perp} \in \ell$ such that $\vartheta$ is strictly decreasing when $p \leq p^{\perp}$, and strictly increasing when $p \geq p^{\perp}$. We prove a similar result when $\ell$ intersects the MEC of $S$.

\section{Preliminaries}

We begin by introducing the notations and definitions used in the rest of the paper. For any two points $a, b$ in the plane, we denote by $[a, b]$ the line segment joining the points $a$ and $b$. For a set of points $Z$ in the plane, the center of the MEC for the point set $Z$ will be denoted by $\mathcal{E}(Z)$ and its radius by $r(Z)$. It is easy to see that the MEC of $Z$ is unique and it will be denoted by $C_{\min }(Z)$.

We now summarize some of the important properties of the MEC, which will be used in the subsequent sections:

Fact 1 The center of the MEC of a set of three points lying on the vertices of a right-angled triangle is the mid-point of the hypotenuse.

Fact 2 An enclosing circle for a set of points $S$ passing through three points of $S$ will be a MEC of $S$ if and only if the triangle formed by the three points is acute-angled or right-angled.

Fact 3 An enclosing circle for a set of points $S$ passing through two points of $S$ will be a MEC of $S$ if and only if the line segment joining the two points is a diameter of the enclosing circle of $S$.

A set $S=\left\{p_{1}, p_{2}, \ldots, p_{n}\right\}$ of $n$ distinct points (sites) in the plane is said to be in general position if no four points of $S$ are co-circular. The farthest-point Voronoi diagram of $S$, to be denoted by $\mathcal{F} \mathcal{V}(S)$, is the subdivision of the plane into $n$ disjoint cells $\left\{F V\left(p_{i}, S\right) \mid p_{i} \in\right.$ $S\}$, excepting the boundaries, where $F V\left(p_{i}, S\right)$ is the set of all points $q \in \mathbb{R}^{2}$ such that 
$d\left(q, p_{i}\right) \geq d\left(q, p_{j}\right)$, for all $i \neq j$. The region $F V\left(p_{i}, S\right)$ is called the farthest-point Voronoi cell of the point $p_{i} \in S$. The farthest-point Voronoi diagram of set of $n$ points in $\mathbb{R}^{2}$ can be computed in $O(n \log n)$ time [6].

The mobile version of the Euclidean 1-center problem addressed in this paper consists of a set $S=\left\{p_{1}, p_{2}, \ldots, p_{n}\right\}$ of $n$ fixed points in the plane and a line $\ell$, not passing through any point of $S$, along which another point moves. Note that for all points $p$ on $\ell$ not lying in the interior of the MEC of $S$, the MEC of $S \cup\{p\}$ always passes through the point $p$. Moreover, for points $p$ on $\ell$ that lie in the interior of the MEC of $S$, the MEC of $S \cup\{p\}$ is the MEC of $S$ itself.

Without loss of generality, choose a coordinate system in which the line $\ell$ coincides with the horizontal axis. Associate with the real number $p$, the point $p=(p, 0)$ on the line $\ell$. Then we define center function $\psi: \mathbb{R} \rightarrow \mathbb{R}^{2}$, as $\psi(p)=\mathcal{E}(S \cup\{p\})$ and the radius function $\vartheta: \mathbb{R} \rightarrow \mathbb{R}$, as $\vartheta(p)=r(S \cup\{p\})$. Therefore, the graph of the center function traces out the locus of the MEC of $S \cup\{p\}$ as the point $p$ moves over $\ell$. For any subset $Q$ of $\mathbb{R}$, let $\psi(Q)=\{\psi(p) \mid p \in Q\}$ and $\vartheta(Q)=\{\vartheta(p) \mid p \in Q\}$.

As mentioned earlier, the motion of the center of the MEC under continuous motion of the points was studied in the context of mobile facility location [9]. Durocher [9] proved that under continuous and bounded velocity motion of the points in the plane the center of the MEC moves continuously. An immediate consequence of this result is the following:

Fact 4 The center function $\psi$ is continuous.

Using these facts, in the following sections we prove that the function $\psi$ is a continuous and piecewise differentiable linear function. Other properties of the center function $\psi$ and the radius function $\vartheta$ are also discussed.

\section{Characterizing the Center Function}

In this section the characteristics of the center function $\psi$ as the point $p=(p, 0)$ movies along the line $\ell$, which is identified with the horizontal axis, are studied. We begin with the following observation when the given set $S$ contains a single point.

Observation 1 If $S$ contains a single point $q=(a, b)$ then the function $\psi$ maps onto the line $y=\frac{b}{2}$ in $\mathbb{R}^{2}$.

Proof. For any point $p=(p, 0)$ on $\ell, \mathcal{E}(S \cup\{p\})$ is the midpoint of the line segment $[q, p]$. As $p$ varies over $\mathbb{R}$ the point $\mathcal{E}(S \cup\{p\})$ varies over the line $y=\frac{b}{2}$ in $\mathbb{R}^{2}$.

Now, we proceed to characterize the locus of the center of the MEC for a set $S=$ $\left\{p_{1}, p_{2}, \ldots, p_{n}\right\}$ of $n$ points in the plane. Denote the circle with the line segment $\left[p_{i}, p_{j}\right]$ as diameter by $C\left(p_{i}, p_{j}\right)$, and the circle passing through $p_{i}$ and $p_{j}$ and tangent to $\ell$ as $C_{\ell}\left(p_{i}, p_{j}\right)$. The point where the circle $C_{\ell}\left(p_{i}, p_{j}\right)$ touches the line $\ell$ will be denoted by $\nu\left(p_{i}, p_{j}\right)=$ $\left(\nu\left(p_{i}, p_{j}\right), 0\right)$. The center of the circles $C\left(p_{i}, p_{j}\right)$ and $C_{\ell}\left(p_{i}, p_{j}\right)$ will be denoted by $c\left(p_{i}, p_{j}\right)$ and $c_{\ell}\left(p_{i}, p_{j}\right)$, respectively.

The following lemma characterizes the center function when the set $S$ contains only two fixed points:

Lemma 1. For $S=\left\{p_{1}, p_{2}\right\}$ the center function $\psi$ is a piecewise differentiable linear function, and each of its differentiable pieces either lies on the perpendicular bisector of $\left[p_{1}, p_{2}\right]$ or on a line parallel to $\ell$. 
Proof. Begin by assuming that the circle $C\left(p_{1}, p_{2}\right)$ does not intersect $\ell$. Let $\ell_{1}\left(p_{1}, p_{2}\right)$ and $\ell_{2}\left(p_{1}, p_{2}\right)$ be the points where the perpendiculars to the line segment $\left[p_{1}, p_{2}\right]$ passing through $p_{1}$ and $p_{2}$, respectively, intersect the line $\ell$ (refer to Fig. 1(a)). Note that for any point $p \in$ $\left[\ell_{1}\left(p_{1}, p_{2}\right), \ell_{2}\left(p_{1}, p_{2}\right)\right]$, the triangle $p p_{1} p_{2}$ is acute-angled or right-angled. Fact 2 then implies that the MEC of $S \cup\{p\}$ is the circumcircle of the triangle $p q r$. Therefore, $\mathcal{E}(S \cup\{p\})$ must lie on the perpendicular bisector of the line segment $\left[p_{1}, p_{2}\right]$. Suppose the perpendicular bisector for the line segment $\left[p_{1}, p_{2}\right]$ intersects the line segments $\left[\ell_{2}\left(p_{1}, p_{2}\right), p_{1}\right]$ and $\left[p_{2}, \ell_{1}\left(p_{1}, p_{2}\right)\right]$ at the points $a\left(p_{1}, p_{2}\right)$ and $b\left(p_{1}, p_{2}\right)$, respectively. Note that, when $p$ is at $\ell_{1}\left(p_{1}, p_{2}\right), \mathcal{E}(S \cup\{p\})$ is at $b\left(p_{1}, p_{2}\right)$, and when $p$ is at $\nu\left(p_{1}, p_{2}\right), \mathcal{E}(S \cup\{p\})=a\left(p_{1}, p_{2}\right)$ (Fact 1). As the point $p$ moves along $\ell$ from $\ell_{1}\left(p_{1}, p_{2}\right)$ to $\nu\left(p_{1}, p_{2}\right), \angle p_{1} p p_{2}$ increases and the radius of the circumcircle of triangle $p_{1} p p_{2}$ decreases. As a result, $\mathcal{E}\left(\left\{p, p_{1}, p_{2}\right\}\right)$ moves towards the point $c_{\ell}\left(p_{1}, p_{2}\right)$. Therefore, for any point $p \in\left[\ell_{1}\left(p_{1}, p_{2}\right), \nu\left(p_{1}, p_{2}\right)\right]$ the function $\psi$ maps onto the line segment $\left[b\left(p_{1}, p_{2}\right), c_{\ell}\left(p_{1}, p_{2}\right)\right]$. Similarly, when $p \in\left[\nu\left(p_{1}, p_{2}\right), \ell_{2}\left(p_{1}, p_{2}\right)\right]$ the function $\psi$ maps onto the line segment $\left[c\left(p_{1}, p_{2}\right), a\left(p_{1}, p_{2}\right)\right]$.

Now, observe that for all points $p$ on $\ell$ with $p<\ell_{1}\left(p_{1}, p_{2}\right), \angle p_{2} p_{1} p$ is greater than $\pi / 2$. So the MEC of $\left\{p_{1}, p_{2}, p\right\}$ is the circle with the lines segment $\left[p, p_{2}\right]$ as diameter. Hence, $\psi(p)$ for all $p<\ell_{1}\left(p_{1}, p_{2}\right)$ is the midpoint of the segment $\left[p, p_{2}\right]$. Therefore, for $p<\ell_{1}\left(p_{1}, p_{2}\right)$ the function $\psi$ maps onto the half-line $\ell_{p_{2}}$, which is the line parallel to $\ell$ emanating from the point $b\left(p_{1}, p_{2}\right)$ lying in the farthest-point Voronoi cell of the point $p_{2}$ (Observation 1$)$. Similarly, for $p>\ell_{2}\left(p_{1}, p_{2}\right)$ the function $\psi$ maps onto the half-line $l_{p_{1}}$, which is the line parallel to $\ell$ emanating from $a\left(p_{1}, p_{2}\right)$ and lying in the farthest-point Voronoi cell of the point $p_{1}$.

Thus, the line $\ell$ can be divided into three intervals, on two of which it maps the function to a line parallel to $\ell$ and on the third, it maps on to the perpendicular bisector of $\left[p_{i}, p_{j}\right]$. The entire function is known to be continuous and on each of these intervals it is differentiable. This completes the proof of the Lemma when $\ell$ does not intersect $C\left(p_{1}, p_{2}\right)$.

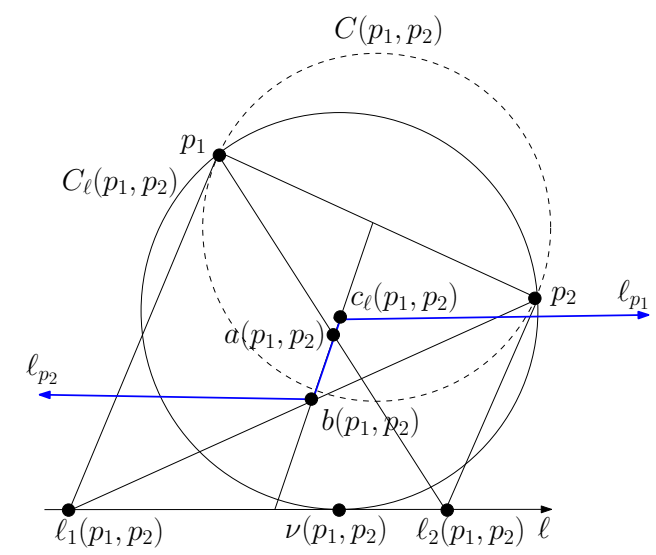

(a)

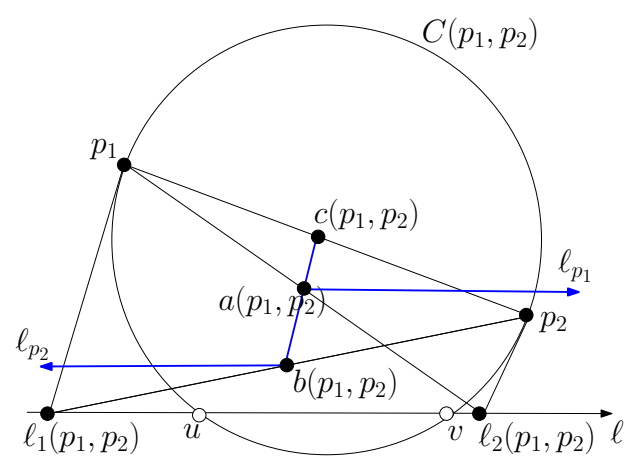

(b)

Fig. 1. Locus of the center of the MEC for two fixed points $p_{1}$ and $p_{2}$ : (a) $C\left(p_{1}, p_{2}\right)$ does not intersect $\ell$, (b) $C\left(p_{1}, p_{2}\right)$ intersects $\ell$.

Next, consider the case where the line $\ell$ intersects $C\left(p_{1}, p_{2}\right)$ at the points $u$ and $v$ (see Fig. 1(b)). For any point $p \in[u, v]$, the MEC of $S \cup\{p\}$ is $C$ itself, and $\psi(p)=c\left(p_{1}, p_{2}\right)$. Therefore, from the same arguments as before it follows that $\psi\left(\left[\ell_{1}\left(p_{1}, p_{2}\right), u\right]\right)=\left[b\left(p_{1}, p_{2}\right), c\left(p_{1}, p_{2}\right)\right]$, $\psi([u, v])=c\left(p_{1}, p_{2}\right)$, and $\psi\left(\left[v, \ell_{2}\left(p_{1}, p_{2}\right)\right]\right)=\left[c\left(p_{1}, p_{2}\right), a\left(p_{1}, p_{2}\right)\right]$. When $p<\ell_{1}\left(p_{1}, p_{2}\right)$ or $p>\ell_{2}\left(p_{1}, p_{2}\right), \psi$ maps onto a line parallel to $\ell$ as described in the previous case. 
Using the above lemma, the center function $\psi$ for a set $S=\left\{p_{1}, p_{2}, \ldots, p_{n}\right\}$ of $n$ points in the plane in general position can be characterized. We denote the farthest point Voronoi vertex equidistant from the site points $p_{i}, p_{j}$ and $p_{k}$ by $v_{i j k}$ and the farthest point Voronoi edge equidistant from the site points $p_{i}$ and $p_{j}$ by $e_{i j}$. Note that the points $a\left(p_{i}, p_{j}\right)$, and $b\left(p_{i}, p_{j}\right)$ lie on the edge $e_{i j}$.

Theorem 1. The center function $\psi$ is a continuous and piecewise differentiable linear function. Moreover, each of the differentiable pieces of function $\psi$ lies either on the boundary of farthest point Voronoi diagram of $S$ or on a line parallel to the line $\ell$.

Proof. From Fact 4 we know that $\psi$ is continuous. For all $p \in \ell$ the MEC of $S \cup\{p\}$ can pass through at most three points of $S$, since points of $S$ are assumed to be in general position. Depending on this we can decompose the line $\ell$ into intervals $\ell_{1}, \ell_{2}, \ldots, \ell_{m}$ with $\ell=\bigcup_{i=1}^{m} \ell_{i}$, such that for all $p \in \ell_{i}$ the MEC of $S \cup\{p\}$ passes through a fixed number of points of $S$. In each interval $\ell_{i}$ one of the following three cases might occur:

Case 1: For all $p \in \ell_{i}$, the MEC of $S \cup\{p\}$ passes through some three points $p_{i}, p_{j}, p_{k} \in$ $S$. Then for all $p \in \ell_{i}$ the point $\mathcal{E}(S \cup\{p\})$ remains fixed at the farthest-point Voronoi vertex $v_{i j k}$.

Case 2: For all $p \in \ell_{i}$, the MEC of $S \cup\{p\}$ passes through two points $p_{i}, p_{j} \in S$. In this situation the problem reduces to the case described in Lemma 1. Thus, $\psi(p)$, for $p \in \ell_{i}$, maps to a subset of the edge $e_{i j}$.

Case 3: For all $p \in \ell_{i}$, the MEC of $S \cup\{p\}$ passes through one point $p_{i} \in S$. Therefore, for $p \in \ell_{i}$, the function $\psi(p)$ lies on the midpoint on the line segment $\left[p_{i}, p\right]$. Therefore, $\psi$ is mapped to a line parallel to $\ell$ emanating from a vertex or an edge of $\mathcal{F} \mathcal{V}(S)$ (Observation $1)$.

From the analysis of these three cases and the continuity of $\psi$, the theorem follows.

The locus of the center of the MEC of a set of 5 fixed points and a moving point is shown in Figure 3. A natural combinatorial problem is to determine the complexity of the number of different linear differentiable pieces of the center function $\psi$.

We begin addressing this problem with the following observations:

Observation 2 Let $p$ and $q$ be two points on the line $\ell$ with $p<q$. Suppose, the MEC of $S \cup\{p\}$ and the $M E C$ of $S \cup\{q\}$ are the circles with diameter $\left[p_{i}, p\right]$ and $\left[p_{i}, q\right]$, respectively, for some fixed $p_{i} \in S$. Then for all points $r \in[p, q]$, the $M E C$ of $S \cup\{r\}$ is the circle with diameter $\left[p_{i}, r\right]$.

Proof. Denote the circles with diameters $\left[p_{i}, p\right]$ and $\left[p_{i}, q\right]$ by $C\left(p_{i}, p\right)$ and $C\left(p_{i}, q\right)$, respectively. Let $f\left(p_{i}\right)$ be the foot of the perpendicular from the point $p_{i}$ on the line $\ell$. These two circles intersect at the points $p_{i}$ and $f\left(p_{i}\right)$, since $\angle p f\left(p_{i}\right) p_{i}=\angle q f\left(p_{i}\right) p_{i}=\pi / 2$ (Fig. $2(\mathrm{a}))$. Observe that all the points in $S$ must lie in the region where the two circles $C\left(p_{i}, p\right)$ and $C\left(p_{i}, q\right)$ overlap. For any point $r \in[p, q]$, the circle $C\left(p_{i}, r\right)$ with diameter $\left[p_{i}, r\right]$ passes through $p_{i}$ and $r$, since $\angle r f\left(p_{i}\right) p_{i}=\pi / 2$, and is also an enclosing circle of $S \cup\{r\}$ (see Fig. $2(\mathrm{a}))$. Fact 3 now implies that the circle $C\left(p_{i}, r\right)$ is the MEC for $S \cup\{r\}$.

The following observation can now be proved similarly (see Fig. 2(b)):

Observation 3 Let $p$ and $q$ be two points on the line $\ell$, with $p<q$. Suppose, for some fixed $p_{i}, p_{j} \in S$, the $M E C$ of $S \cup\{p\}$ and the $M E C$ of $S \cup\{q\}$ are the circumcircles of triangles $p_{i} p_{j} p$ and $p_{i} p_{j} q$, respectively. Then for all points $r \in[p, q]$, the $M E C$ of $S \cup\{r\}$ is the circumcircle of triangle $p_{i} p_{j} r$. 


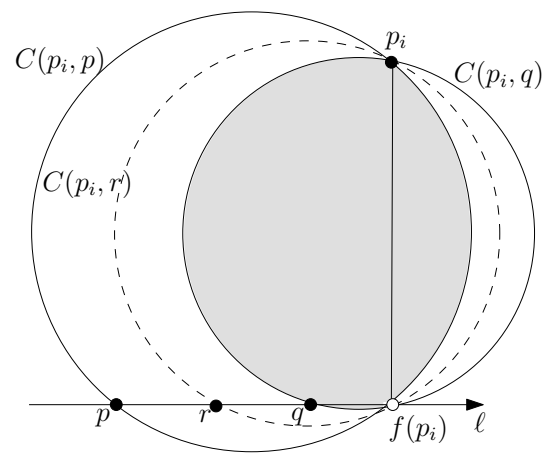

(a)

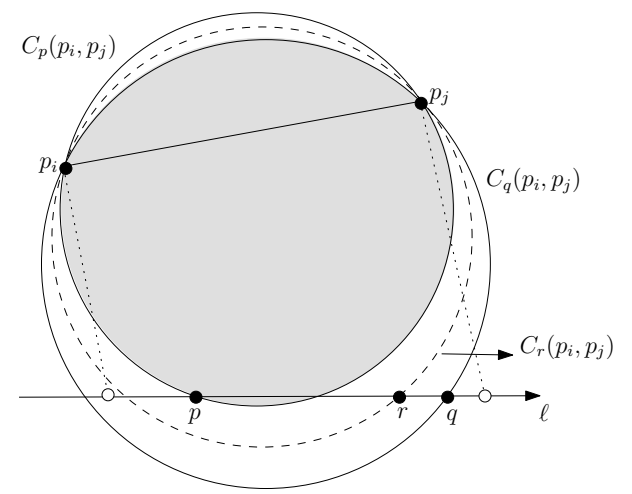

(b)

Fig. 2. (a) Illustration for the proof of Observation 2, (b) Illustration for the proof of Observation 3.

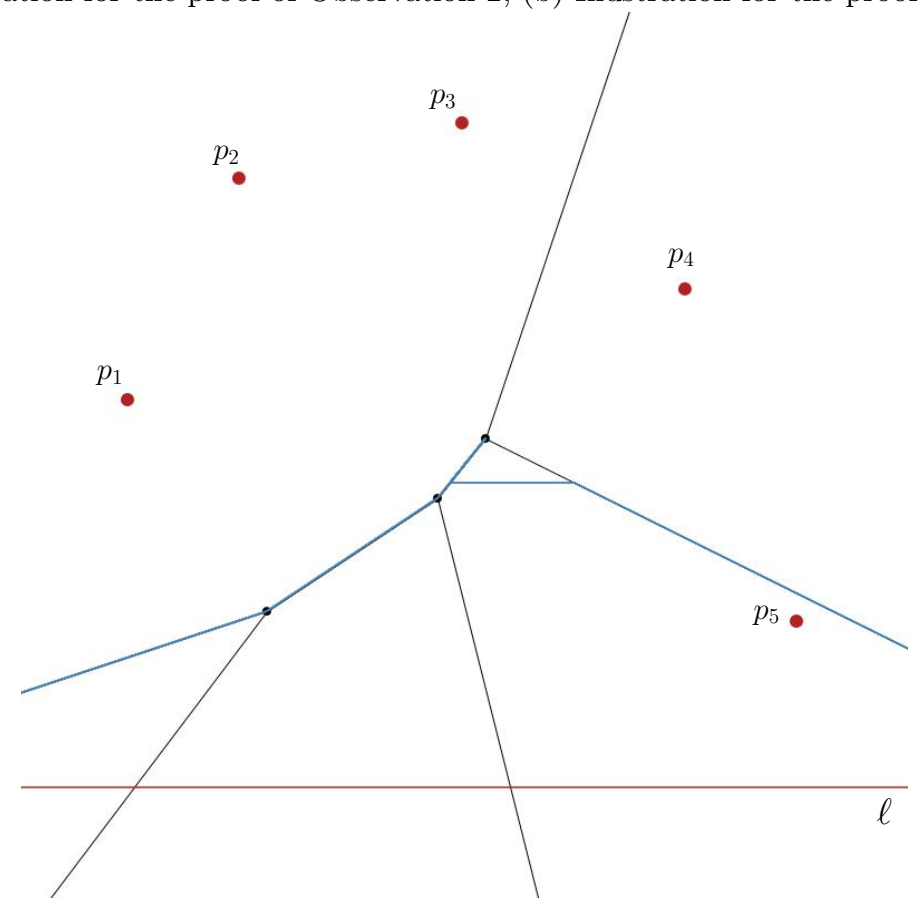

Fig. 3. Locus of the center of the MEC of a set of 5 fixed points and a point moving along the line $\ell$.

Using these two observations we now prove the following result about the number of differentiable pieces in $\psi$ :

Theorem 2. The function $\psi$ can have at most $O(n)$ differentiable pieces.

Proof. Theorem 1 implies that the line $\ell$ can be decomposed into intervals $\ell_{1}, \ell_{2}, \ldots, \ell_{m}$ such that $\ell=\bigcup_{i=1}^{m} \ell_{i}$, and for all points $p \in \ell_{i}, \mathcal{E}(S \cup\{p\})$ is either on a line parallel to $\ell$, or on some edge of $\mathcal{F} \mathcal{V}(S)$. Observation 2 implies the number of line segments in $\psi$ that are parallel to $\ell$ is $O(|S|)=O(n)$. Similarly, Observation 3 implies that for any fixed edge $e_{i j}$ of $\mathcal{F} \mathcal{V}(S)$ there is at most one interval $\ell_{i j}$ on $\ell$ such that whenever $p \in \ell_{i j}$, the function $\psi(p)$ lies on $e_{i j}$. In every such interval, the function $\psi$ can have at most 2 differentiable pieces. Therefore, the number differentiable pieces in $\psi$ lying on $\mathcal{F} \mathcal{V}(S)$ is at most twice the number of edges in $\mathcal{F V}(S)$. Since the number of edges in $\mathcal{F V}(S)$ is $O(n)$ [6], the result follows. 


\section{Computation of the Center Function}

We now present the algorithm for computing the center function $\psi$, given the farthest-point Voronoi diagram of $S$. In order to find the function $\psi$ explicitly, the line sweep technique is used. The points on $\psi$ where the function changes from one line segment to the another are considered as event points.

Let $p_{\min }$ be the point in $S$ having the minimum $x$-coordinate. Suppose $p_{\infty}$ is the point on $\ell$ with the least $x$-coordinate such that for all $p>p_{\infty}$, the MEC of $S \cup\{p\}$ is the circle with $\left[p, p_{\min }\right]$ as diameter. Moreover, for any point $p_{i}=(a, b) \in S$ denote the line $y=\frac{b}{2}$ by $\ell\left(p_{i}\right)$. The algorithm can now be described as follows:

We begin by computing $p_{\min }$ and the intersection point $\delta$ between $\ell\left(p_{\min }\right)$ and the boundary of $F V\left(p_{\min }, S\right)$. We insert $\delta$ into the event queue. After initialization, depending upon the type of the present event point and the nature of the locus, the next event point is determined as follows:

Case 1: The current locus is at $\ell\left(p_{i}\right)$ and it intersects with the Voronoi edge $e_{i j}$. Compute the intersection point $\delta$ between $\ell\left(p_{j}\right)$ and $e_{i j}$. The next event point is either $\delta$ or a farthest point voronoi vertex $v_{i j k}$, depending upon which one is nearer to the current event point.

Case 2: The current locus is a segment of Voronoi edge $e_{i j}$ and the current event point is the intersection point of $e_{i j}$ and $\ell\left(p_{i}\right)$. Then, the next event point is the intersection point between $\ell\left(p_{i}\right)$ and the boundary of $F V\left(p_{i}, S\right)$.

Case 3: The current event point is a vertex $v_{i j k}$. We compute the edge $e_{i j}$ that $\psi$ follows hereafter. The next event point can be computed as in Case 1.

In the third case described above, we need to find the edge that $\psi$ will follow when the current event point is at some vertex $v_{i j k}$. In the following lemma we show that this can be done in constant time.

Lemma 2. If the sweep line reaches a farthest point Voronoi vertex $v_{i j k}$ by an edge then it takes constant time to locate the next edge.

Proof. Let $p$ be the point on $\ell$ such that the center of the MEC of $S \cup\{p\}$, say $C_{p}$, is at a vertex $v_{i j k}$ of $\mathcal{F} \mathcal{V}(\mathcal{S})$. Consider the diameter of $C_{p}$ passing through $p$. As $C_{p}$ is minimum enclosing, exactly two of the three points $p_{i}, p_{j}$ and $p_{k}$ will be on the one side of the diameter through $p$. Without loss of generality, assume that these two points are $p_{j}$ and $p_{k}$ (see figure $4(\mathrm{a}))$. As $\angle p_{j} p_{k} p$ is not acute, for any point $p_{\epsilon}$ sufficiently close to $p, \angle p_{j} p_{k} p_{\epsilon}$ is also not acute. Hence, depending on the direction of the sweep line either it will reach vertex $v_{i j k}$ by $e_{i j}$ and leave by $e_{i k}$ or vice versa.

Using this lemma we can now prove the following theorem:

Theorem 3. The algorithm described above for computing the center function $\psi$ runs in $O(n)$ time.

Proof. Note that $p_{\min }$ and $p_{\infty}$ can be computed in linear time. Lemma 2 ensures that when at a vertex, the next edge that $\psi$ follows can be determined in constant time. Now, to find the intersection point of the $\ell\left(p_{i}\right)$, for some $p_{i} \in S$, with the boundary of $F V\left(p_{i}, S\right)$, we need $O\left(\left|E_{i}\right|\right)$ time, where $\left|E_{i}\right|$ is the number of edges bounding $F V\left(p_{i}, S\right)$. Since each edge is in the boundary of at most two farthest point Voronoi cells and $\psi$ can lie on each 
$\ell\left(p_{i}\right)$ at most once (Lemma 2), the process repeated over all points in $S$ takes at most $O(2|E|)=O(n)$ time, where $|E|$ is the number of edges in $\mathcal{F} \mathcal{V}(S)$. Therefore, given $\mathcal{F} \mathcal{V}(S)$, the center function $\psi$ can be computed in $O(n)$ time.

Remark: A polyline of complexity $m$ is a connected series of $m$ line segments. Observe that all the above results can be generalized to the case where the point moves along a polyline $P_{m}$ of complexity $m$. Given a set $S$ of $n$ points and a polyline $P_{m}$ the locus of the MEC of $S \cup\{p\}$ as $p$ varies over $P_{m}$ is a continuous and piecewise differentiable linear curve, and each of its differentiable piece lies either on the boundary of the farthest-point Voronoi diagram of $S$, or on a line segment parallel to a line segment of $P_{m}$. Moreover, as in Theorem 2 the locus can have at most $O(m n)$ differentiable pieces and thus can be computed easily in $O(m n)$ time, given the farthest-point Voronoi diagram of $S$.

\section{Properties of the Radius Function}

The center function traces the path of the center of the MEC as a point moves along the line $\ell$. Analogously, we can analyze the radius of the MEC as the point moves along the line $\ell$. In this section, the properties of the radius of the MEC of $S \cup\{p\}$ as the point $p$ moves along the line $\ell$, is studied. For a set $S$ of $n$ points $\left\{p_{1}, p_{2}, \ldots, p_{n}\right\}$ in general position, the radius function $\vartheta: \mathbb{R} \rightarrow \mathbb{R}$ is defined as $\vartheta(p)=r(S \cup\{p\})$, where $p=(p, 0) \in \ell$. Note that as the center function $\psi$ is continuous, the radius of the MEC of $S \cup\{p\}$ changes continuously as the point $\mathcal{E}(S \cup\{p\})$ moves along the graph of the function $\psi$. This immediately implies that the function $\psi$ is continuous.

The MEC of $S$ will be denoted by $C(S)$, and depending on whether the line $\ell$ intersects $C(S)$ two cases are considered.

\section{$5.1 C(S) \cap \ell=\emptyset$}

Let $p^{\perp}$ be the point on $\ell$ such that the MEC of $S \cup\left\{p^{\perp}\right\}$ is tangent to $\ell$ at the point $\ell$. Note that there is exactly one such point on $\ell$ satisfying this property. Denote the minimum enclosing circle of $S \cup\left\{p^{\perp}\right\}$ by $C_{p^{\perp}}$. We shall prove that in this case the radius function $\vartheta$ has a unique minimum at the point $p^{\perp}$. In fact, $\vartheta$ is strictly decreasing when $p \leq p^{\perp}$, and strictly increasing when $p \geq p^{\perp}$.

For any three points $a, b, c \in \mathbb{R}^{2}$, define $\mathcal{H}(a b, c)$ to be the open half-space bounded by the line passing through the points $a$ and $b$, and containing the point $c$. We now have the following lemma:

Lemma 3. For all points $p \in \ell$ the $M E C$ of $S \cup\{p\}$ either passes through or contains the point $p^{\perp}$.

Proof. Suppose there exists a point $p=(p, 0)$ on $\ell$ such the MEC of $S \cup\{p\}$ does not contain $\ell$. Without loss of generality assume that $p \leq p^{\perp}$. Denote the minimum enclosing circle of $S \cup\{p\}$ by $C_{p}$, and $q, q^{\prime}$ be the points where the circles $C_{p^{\perp}}$ and $C_{p}$ intersect (refer to Fig.4(b)). Let $p_{0}^{\perp}$ be the point on the boundary of $C_{p^{\perp}}$ diametrically opposite to $p^{\perp}$. If $q$ is in the open half-plane $\mathcal{H}\left(p^{\perp} p_{0}^{\perp}, p\right)$, then no point of $S$ lies on the boundary of the circle $C_{p^{\perp}}$ on one side of the diameter $p^{\perp} p_{0}^{\perp}$. This contradicts the fact that $C_{p^{\perp}}$ is the MEC of $S \cup\left\{p^{\perp}\right\}$ (Fact 2). Let $p_{0}$ be the diametrically opposite point of $p_{0}$. Now, if $q$ is in the open half-plane $\mathcal{H}\left(p p_{0}, p^{\perp}\right)$ then there will be no point of $S$ on the boundary of $C_{p}$ on one side of 


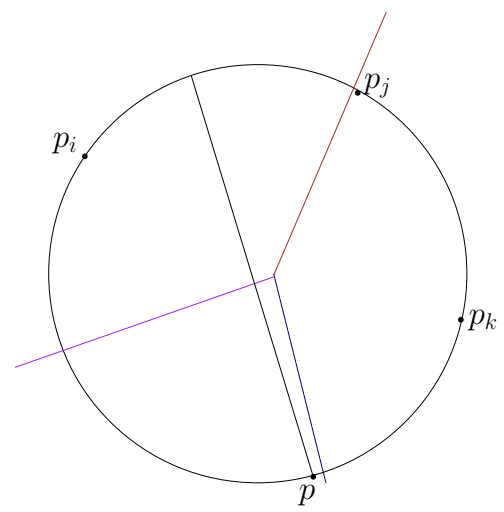

(a)

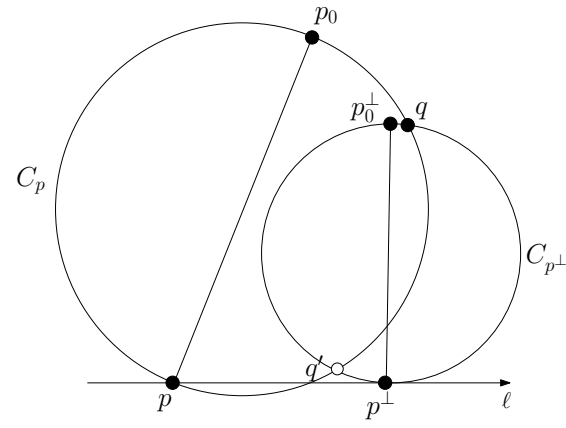

(b)

Fig. 4. (a) Illustration for the proof of Lemma 2, (b) Illustration for the proof of Lemma 3.

a diameter $p p_{0}$. Therefore, $q \notin \mathcal{H}\left(p^{\perp} p_{0}^{\perp}, p\right) \cup \mathcal{H}\left(p p_{0}, p^{\perp}\right)$. This implies that the circle $C_{p}$ with diameter $\left[p, p_{0}\right]$ always contains the point $p^{\perp}$, which contradicts our initial assumption, and the result follows.

Proposition 1. If $C(S) \cap \ell=\emptyset$, the radius function $\vartheta$ is strictly decreasing when $p \leq p^{\perp}$, and strictly increasing when $p \geq p^{\perp}$.

Proof. Consider any two points $p_{a}<p_{b}<p^{\perp}$ on $\ell$, and denote the MEC of $S \cup\left\{p_{a}\right\}$ by $C_{a}$ and the MEC of $S \cup\left\{p_{b}\right\}$ by $C_{b}$. As $C_{a}$ contains $p^{\perp}$ (Lemma 3), it also contains the line segment $\left[p_{a}, p_{b}\right]$. Therefore, $C_{a}$ is an enclosing circle for $S \cup\left\{p_{b}\right\}$. This implies that $\vartheta\left(p_{a}\right)=r\left(S \cup\left\{p_{a}\right\}\right)>r\left(S \cup\left\{p_{b}\right\}\right)=\vartheta\left(p_{b}\right)$, where the inequality is strict because the MEC of $S \cup\left\{p_{b}\right\}$ always passes through the point $p_{b}$ and it is unique. Note that if $p_{a}<p_{b}=p^{\perp}$ then $C_{a}$ contains the line segment $\left[p_{a}, p^{\perp}\right]$. However, the MEC $C_{p^{\perp}}$ of $S \cup\left\{p^{\perp}\right\}$ is tangent to $\ell$ at the point $p^{\perp}$. The uniqueness of the MEC again implies that $\vartheta\left(p_{a}\right)>\vartheta\left(p^{\perp}\right)$. This proves that the radius function $\vartheta$ is strictly decreasing when $p \leq p^{\perp}$.

Similarly, we can show that the radius function $\vartheta$ is strictly increasing when $p \geq p^{\perp}$.

\section{$5.2 C(S) \cap \ell \neq \emptyset$}

In this section, we consider the case where the MEC of $S(C(S))$ intersects the line $\ell$. Let $C(S)$ intersects $\ell$ at points $p^{\prime}$ and $p^{\prime \prime}$, with $p^{\prime}<p^{\prime \prime}$.

Analogous to Lemma 3 , the following result can be proved similarly.

Lemma 4. For all points $p<p^{\prime}$ the $M E C$ of $S \cup\{p\}$ either passes through or contains the point $p^{\prime}$ and for all point $p>p^{\prime \prime}$ the $M E C$ of $S \cup\{p\}$ either passes through or contains the point $p^{\prime \prime}$.

Using this lemma, we prove the following proposition for the case where the line $\ell$ does not intersect the MEC of $S$.

Proposition 2. If $C(S) \cap \ell=\emptyset$, the radius function $\vartheta$ is strictly decreasing when $p<p^{\prime}$, and strictly increasing when $p>p^{\prime \prime}$ and constant when $p^{\prime} \leq p \leq p^{\prime \prime}$.

Proof. Consider any two points $p_{a}<p_{b}<p^{\prime}$ on $\ell$, and denote the MEC of $S \cup\left\{p_{a}\right\}$ by $C_{a}$ and the MEC of $S \cup\left\{p_{b}\right\}$ by $C_{b}$. As $C_{a}$ contains $p^{\prime}$ (Lemma 3), it also contains the line segment $\left[p_{a}, p_{b}\right]$. Therefore, $C_{a}$ is an enclosing circle for $S \cup\left\{p_{b}\right\}$. This implies that 
$\vartheta\left(p_{a}\right)=r\left(S \cup\left\{p_{a}\right\}\right)>r\left(S \cup\left\{p_{b}\right\}\right)=\vartheta\left(p_{b}\right)$, where the inequality is strict because the MEC of $S \cup\left\{p_{b}\right\}$ always passes through the point $p_{b}$ and it is unique. This proves that the radius function $\vartheta$ is strictly decreasing when $p<p^{\prime}$.

Similarly, we can show that the radius function $\vartheta$ is strictly increasing when $p>p^{\prime \prime}$.

Finally, when $p^{\prime} \leq p \leq p^{\prime \prime}$, the point $p$ lies on the chord $\left[p^{\prime}, p^{\prime \prime}\right]$ of the MEC of $S$, and the MEC of $S \cup\{p\}$ is same as the MEC of $S$. Hence, for all $p \in\left[p^{\prime}, p^{\prime \prime}\right], \vartheta(p)=r(S)$.

Acknowledgement: The authors are indebted to the anonymous referees for valuable comments which improved the presentation of the paper.

\section{References}

1. P. K. Agarwal and S. Har-Peled, Maintaining approximate extent measures of moving points, In Proceedings of the Symposium on Discrete Algorithms, 148-157, ACM Press, 2001.

2. P. K. Agarwal, M. Sharir, and S. Toledo, An efficient multi-dimensional searching technique and its applications, Technical Report CS-1993-20, Department of Computer Science, Duke University, 1993.

3. S. Bitner, Y. K. Cheung, and O. Daescu, Minimum separating circle for bichromatic points in the plane, Proc. 7th International Symposium on Voronoi Diagrams (ISVD), 50-55, 2010.

4. S. Bespamyatnikh, B. Bhattacharya, D. Kirkpatrick, and M. Segal, Mobile facility location, In Proceedings of the International ACM Workshop on Discrete Algorithms and Methods for Mobile Computing and Communications, Vol. 4, 46-53, 2000.

5. S. Bereg, B. Bhattacharya, D. Kirkpatrick, and M. Segal, Competitive algorithms for mobile centers, Mobile Networks and Applications, Vol. 11(2), 177-186, 2006.

6. M. de Berg, M. Van Kreveld, M. Overmarse and O. Schwarzkopf, Computational Geometry Algorithms and Applications, Springer-Verlag, 1997.

7. B. Chazelle and J. Matoušek, On linear-time deterministic algorithms for optimization problems in fixed dimensions, Journal of Algorithms, Vol. 21, 579-597, 1996.

8. Y. K. Cheung, O. Daescu, and M. Zivanic, Kinetic red-blue minimum separating circle, Proc. 5th International Conference on Combinatorial Optimization and Applications (COCOA), LNCS 6831, 448-463, 2011.

9. S. Durocher, Geometric Facility Location under Continuous Motion: Bounded-Velocity Approximations to the Mobile Euclidean k-Centre and k-Median Problems, Ph. D. Thesis, University of British Columbia, Canada, 2006.

10. J. Gao, L. J. Guibas, J. Hershberger, L. Zhang, and A. Zhu, Discrete mobile centers, Discrete and Computational Geometry, 30(1), 45-65, 2003.

11. N. Megiddo, Linear-time algorithms for linear programming in $\mathbb{R}^{3}$ and related problems, SIAM Journal on Computing, Vol. 4, 759-776, 1983.

12. J. J. Sylvester, A question in the geometry of situation, Quarterly Journal of Mathematics, 1:79, 1857.

13. E. Welzl, Smallest enclosing disks (balls and ellipsoids), In H. Maurer (Ed.), New Results and New Trends in Computer Science, LNCS 555, 359-370, 1991. 